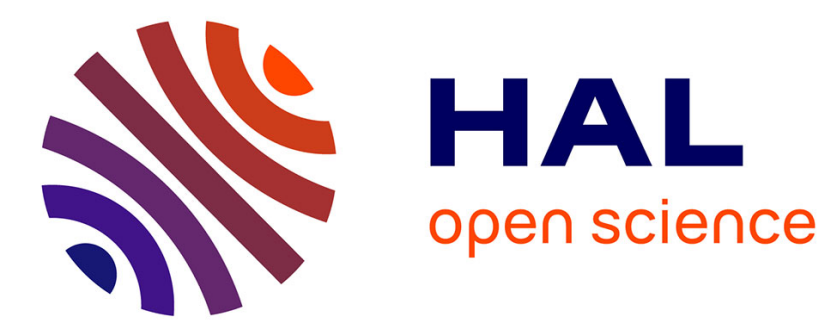

\title{
Le troc en Russie. Un problème de liquidité ou de solvabilité?
}

Mathilde Maurel, Sophie Brana

\section{To cite this version:}

Mathilde Maurel, Sophie Brana. Le troc en Russie. Un problème de liquidité ou de solvabilité?. Revue Economique, 2000, 51 (3), pp.659-669. hal-01011194

\section{HAL Id: hal-01011194 \\ https://hal-sciencespo.archives-ouvertes.fr/hal-01011194}

Submitted on 23 Jun 2014

HAL is a multi-disciplinary open access archive for the deposit and dissemination of scientific research documents, whether they are published or not. The documents may come from teaching and research institutions in France or abroad, or from public or private research centers.
L'archive ouverte pluridisciplinaire HAL, est destinée au dépôt et à la diffusion de documents scientifiques de niveau recherche, publiés ou non, émanant des établissements d'enseignement et de recherche français ou étrangers, des laboratoires publics ou privés. 


\title{
Persée
}

http://www.persee.fr

\section{Le troc en Russie. Un problème de liquidité ou de solvabilité ?}

\author{
Mathilde Maurel;Sophie Brana \\ Revue économique, Année 2000, Volume 51, Numéro 3 \\ p. 659 - 669
}

Voir l'article en ligne

\section{Avertissement}

L'éditeur du site " PERSEE » - le Ministère de la jeunesse, de l'éducation nationale et de la recherche, Direction de l'enseignement supérieur, Sous-direction des bibliothèques et de la documentation - détient la propriété intellectuelle et les droits d'exploitation. A ce titre il est titulaire des droits d'auteur et du droit sui generis du producteur de bases de données sur ce site conformément à la loi n`98-536 du 1 er juillet 1998 relative aux bases de données.

Les oeuvres reproduites sur le site «PERSEE » sont protégées par les dispositions générales du Code de la propriété intellectuelle.

Droits et devoirs des utilisateurs

Pour un usage strictement privé, la simple reproduction du contenu de ce site est libre.

Pour un usage scientifique ou pédagogique, à des fins de recherches, d'enseignement ou de communication excluant toute exploitation commerciale, la reproduction et la communication au public du contenu de ce site sont autorisées, sous réserve que celles-ci servent d'illustration, ne soient pas substantielles et ne soient pas expressément limitées (plans ou photographies). La mention Le Ministère de la jeunesse, de l'éducation nationale et de la recherche, Direction de l'enseignement supérieur, Sous-direction des bibliothèques et de la documentation sur chaque reproduction tirée du site est obligatoire ainsi que le nom de la revue et- lorsqu'ils sont indiqués - le nom de l'auteur et la référence du document reproduit.

Toute autre reproduction ou communication au public, intégrale ou substantielle du contenu de ce site, par quelque procédé que ce soit, de l'éditeur original de l'oeuvre, de l'auteur et de ses ayants droit.

La reproduction et l'exploitation des photographies et des plans, y compris à des fins commerciales, doivent être autorisés par l'éditeur du site, Le Ministère de la jeunesse, de l'éducation nationale et de la recherche, Direction de l'enseignement supérieur, Sous-direction des bibliothèques et de la documentation (voir http://www.sup.adc.education.fr/bib/ ). La source et les crédits devront toujours être mentionnés. 


\title{
Le troc en Russie
}

\section{Un problème de liquidité ou de solvabilité ?}

\author{
Sophie Brana* \\ Mathilde Maurel ${ }^{* *}$
}

\begin{abstract}
Le troc en Russie est engendré par une contrainte de liquidité qui diffère selon la situation économique des entreprises. Pour celles dont la situation financière est relativement bonne mais qui sont confrontées à des problèmes d'anti-sélection et n'ont pas accès au crédit bancaire, il agit comme un substitut au crédit bancaire de court terme. En revanche, les firmes endettées l'utilisent comme moyen de financement externe, évitant ainsi des restructurations coûteuses.
\end{abstract}

\section{BARTER IN RUSSIA: A MATTER OF LIQUIDITY OR A MATTER OF SOLVENCY?}

Barter in Russia can be explained by firms liquidity constraint: it is strongly correlated with financial tightness. However a micro-economic analysis reveals that the rationale behind this liquidity constraint is different according to the firm situation. For firms in a good economic situation, but faced with adverse selection problem and having no access to bank credit, barter acts as a substitute for short term credit. While for indebted firms, barter, in the same way as external finance, is a way of avoiding costly restructuring.

Classification JEL : E41, E51, P24

Alors qu'il représentait moins de $5 \%$ des ventes industrielles en 1992, le troc a connu depuis une expansion rapide et continue et concerne aujourd'hui près de $55 \%$ des ventes. Inconnu dans la plupart des autres économies en transition, il n'est qu'une composante parmi d'autres - la dollarisation, l'accumulation d'arriérés fiscaux, interentreprises ou salariaux, les veksels, les bons de compensation - du processus de démonétisation de l'économie russe.

Le recours au troc peut procéder de deux logiques différentes. Il peut tout d'abord provenir d'un choix stratégique. Les entreprises préfêrent éviter les transactions monétaires soit parce que la monnaie légale ne remplit pas correctement ses fonctions (Poser [1998]), soit parce que le troc procure un certain nombre d'avantages, notamment fiscaux (Ickes et al. [1997] ; Hendlez et al. [1997]). Une autre explication possible repose sur le caractère contraint du recours au troc, dans un contexte de pénurie de liquidités. Le troc peut ainsi être

* LARefi, Université Montesquieu-Bordeaux IV, avenue Léon-Duguit, 33608 Pessac. E-mail : brana@montesquieu.u-bordeaux.fr

** ROSES (CNRS), Université Paris I, Maison des Sciences économiques, 106112 boulevard de l'Hôpital, 75013 Paris. (E-mail : Maurelm@univ-paris1.fr) et CEPR. 
perçu comme une réponse des entreprises aux contraintes de liquidité, leur permettant de maintenir leur niveau d'activité (Linz et Krueger [1998]).

\section{LES MOTIVATIONS STRATÉGIQUES DU TROC}

\section{Le troc comme moyen d'évasion fiscale}

Johnson, Kaufmann et Shleifer [1997] mettent en évidence, dans un modèle simple, les raisons qui poussent les firmes à choisir entre l'économie formelle et l'économie informelle. Les entreprises entrent sur le marché officiel si les gains dépassent les coûts. Le gouvernement, sur ce marché, fournit des biens publics qui augmentent la productivité des entreprises, mais impose en retour des taxes et des réglementations. Ainsi, plus les effets de réseau potentiels seront faibles, plus grande sera l'incitation à rester sur le secteur informel. De même, plus les taxes, prélevées uniquement quand l'entreprise reçoit un paiement monétaire pour ses livraisons, seront élevées, plus l'incitation à l'évasion fiscale sera grande. Pour Gaddy et Ickes [1998], le motif fiscal serait l'explication principale du troc en Russie.

Cette explication n'est pourtant pas convaincante pour au moins deux raisons. Le recours au troc suppose tout d'abord l'existence d'une «double coïncidence » des désirs d'échange de la part des agents participant à la transaction, ce qui entraîne des coûts liés au temps de recherche ainsi que des coûts de stockage, et d'un réseau d'échanges qui peut s'avérer très coûteux (Linz et Krueger [1998]). Dès lors, il n'est pas certain que les bénéfices du troc, liés à l'évasion fiscale, soient supérieurs aux coûts qui y sont associés.

Ensuite, le paiement en «liquide» est un moyen plus simple de ne pas déclarer ses revenus. Dans les pays occidentaux, c'est le moyen le plus répandu de fraude fiscale. En Russie, la mission de collecte fiscale est assurée par les banques qui sont tenues d'informer les autorités concernées de tous les ordres de paiement reçus et qui doivent débiter les comptes des agents ayant une dette fiscale. Si, dans ce contexte, les entreprises ont effectivement une incitation forte à ne pas effectuer leurs paiements via le système bancaire, elles peuvent très bien effectuer leurs paiements en liquide.

Le Russian Economic Barometer (REB) présente, en les hiérarchisant, les raisons qui poussent les entreprises à utiliser le troc. Le désir de contourner la législation fiscale est présent pour $20 \%$ d'entre elles, mais il est loin de constituer le facteur dominant (Aukutsionek [1998]). Commander et Mumssen [1998] mentionnent d'ailleurs que la plupart des entreprises pensent que le troc augmente leur facture fiscale, en partie parce que les prix de troc sont presque toujours supérieurs aux prix monétaires. Dans leur étude, seules $13 \%$ des entreprises mentionnent un motif fiscal comme raison importante du troc ${ }^{1}$.

1. Les deux sont pourtant liés puisque près de la moitié des paiements fiscaux sont non monétaires. 


\section{Le troc comme substitut à la monnaie déficiente}

Le troc peut se développer lorsque la monnaie n'est plus capable de remplir ses fonctions de moyen d'échange, d'unité de compte et de réserve de valeur (Poser [1998]). Les défaillances du système de paiement, notamment les délais de paiement, font peser un coût d'opportunité sur les entreprises, compte tenu du contexte inflationniste, et favorisent les risques de crédit et de liquidité. Le recours au troc, en garantissant le paiement immédiat, permet d'éviter les problèmes de «hold-up » liés à l'échec du système de paiement en termes de coordination et de règlement. Il permet également, dans un contexte de forte inflation, d'éviter le coût d'opportunité lié à la détention d'encaisses liquides ${ }^{1}$. Le développement du troc en Russie a ainsi d'abord été perçu comme une réaction naturelle au contexte de forte inflation qui a suivi la période de libéralisation des prix. Mais sur le long terme, avant et après la stabilisation, la corrélation entre les variables de troc et de taux d'inflation apparaît négative.

\begin{tabular}{c|c|c|c}
\hline Troc & Troc & $\begin{array}{c}\text { Taux d'inflation } \\
\text { (prix à la } \\
\text { consommation) }\end{array}$ & $\begin{array}{c}\text { Taux d'inflation } \\
\text { (prix à la } \\
\text { production) }\end{array}$ \\
\hline $\begin{array}{c}\text { Taux d'inflation } \\
\text { (prix à la consommation) } \\
\begin{array}{c}\text { Taux d'inflation } \\
\text { (prix à la production) }\end{array}\end{array}$ & $-0,69$ & 1 & 1 \\
\hline
\end{tabular}

Sources des données : Russian Economic Trend et Russian Economic Barometer (1992-1 ; 1998-7, données mensuelles).

Si le troc a bien augmenté au moment de la libéralisation des prix, la mise en place effective de politiques de stabilisation n'a pas inversé la tendance à l'augmentation de la part des transactions effectuées sous forme de troc. L'hypothèse que ce dernier est un substitut à une monnaie se dépréciant n'est donc pas validée. La mise en évidence d'une corrélation négative entre le troc et l'inflation peut, au contraire, suggérer l'existence d'une contrainte de liquidité.

\section{LE TROC, RÉPONSE À UNE CONTRAINTE DE LIQUIDITÉ}

L'existence d'une corrélation négative entre le taux d'inflation et l'évolution du troc semble indiquer l'existence d'une contrainte de liquidité. Celle-ci pourrait provenir d'un resserrement de politique monétaire qui obligerait les entre-

1. Enginer et Bernhardt [1991] construisent un modèle théorique dans lequel la monnaie est en concurrence avec le troc. Ils montrent que le troc émerge s'il existe une double coïncidence des besoins et si les échanges monétaires sont limités par la contrainte de liquidité. Il existe dans leur modèle un taux d'inflation unique en dessous duquel le seul moyen de paiement est la monnaie, et au-dessus duquel la monnaie n'a aucune valeur. Voir également Hayashi et Matsui [1994]. 
prises à développer des substituts monétaires. Par exemple, Alfanderi et Schaffer [1996], observant l'existence d'un lien étroit et négatif entre l'évolution du taux d'inflation et celle des arriérés, attribuent la forte croissance de ces derniers à la mise en place d'une politique monétaire restrictive à partir de 1992.

Nous testons l'hypothèse d'une relation entre troc et contrainte de liquidité au niveau de la firme à partir de la variable goodfin, qui indique le pourcentage d'entreprises qui considèrent être dans une bonne situation financière. L'influence de la politique monétaire est mesurée par le taux de liquidité de l'économie, le niveau réel du taux de refinancement et le taux d'inflation. On teste l'hypothèse selon laquelle le ralentissement de la création monétaire (de l'inflation) ou la hausse du taux de refinancement expliquent, ou « causent », au sens de Granger, le recours croissant au troc.

Tableau 1. Corrélations et tests de causalité entre le troc et quelques indicateurs financiers

\begin{tabular}{|c|c|c|c|}
\hline & \multirow{2}{*}{$\begin{array}{l}\text { Corrélation } \\
\text { avec le troc }\end{array}$} & \multicolumn{2}{|c|}{$\begin{array}{l}\text { Tests de causalité à la Granger } \\
\text { (Statistique de Fisher } \\
\text { à } 6 \text { degrés de liberté) }\end{array}$} \\
\hline & & $\begin{array}{c}\text { Ensemble } \\
\text { de la période } \\
1992(1)-1998(1)\end{array}$ & $\begin{array}{c}\text { Début } \\
\text { de la période } \\
\text { 1992(1)-1996(1) }\end{array}$ \\
\hline Situation financière (goodfin) .......... & $-59 \%$ & $2,32 *$ & $3,49 *$ \\
\hline Taux d'inflation & $-82 \%$ & 0,71 & 0,76 \\
\hline Taux réel de refinancement.............. & $41 \%$ & 1,7 & $3,47 *$ \\
\hline Rendement réel sur GKO ................... & $71 \%$ & 0,66 & 0,82 \\
\hline Taux de liquidité ................................... & $49 \%$ & 1,04 & 0,71 \\
\hline
\end{tabular}

Les tests de causalité à la Granger ont été effectués sur variables stationnaires (les variables I(1) ont été différenciées). Les données sont mensuelles.

*, ** indique une significativité à $95 \%, 99 \%$.

Sources des données : Russian Economic Trend et Russian Economic Barometer.

Seules deux relations de causalité sont mises en évidence : le troc augmente avec le niveau des taux d'intérêt réels et quand la situation financière des entreprises se dégrade. Le graphique 1 illustre bien ces deux relations.

L'interdépendance de ces trois variables est renforcée par l'estimation d'équations de long terme. Les variables étant stationnaires en différence, nous recherchons l'existence de vecteurs coïntégrants, en utilisant la méthode du maximum de vraisemblance de Johansen.

Le tableau 2 indique l'existence d'un seul vecteur coïntégrant. La relation de long terme associée s'écrit :

$$
\text { troc }=-0,52 \text { goodfin }+0,14 \text { taux d'intérêt }
$$




\section{Graphique 1. Évolution du troc et de la situation financière}

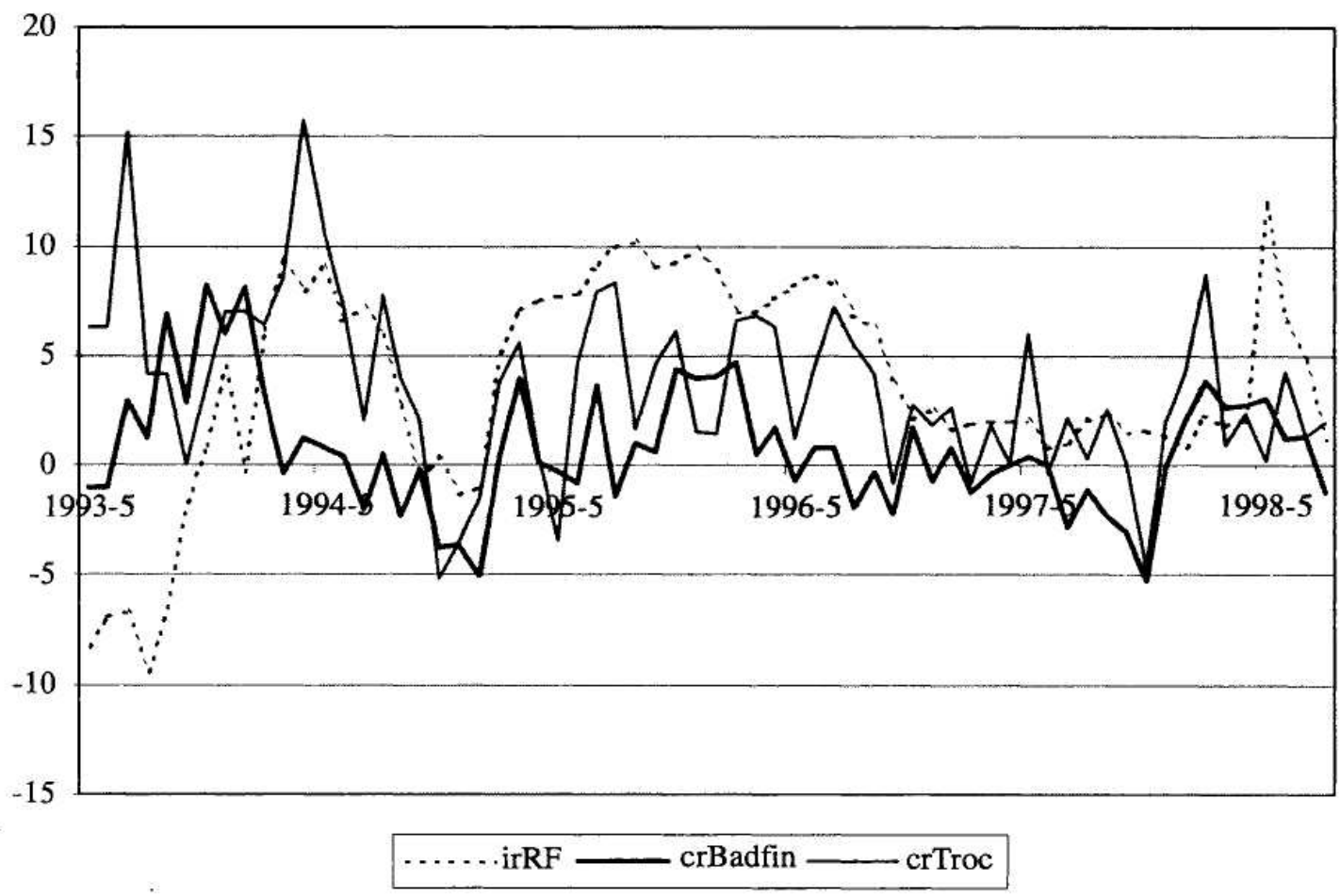

avec irRF, le taux d'intérêt réel de refinancement ; crBadfin, la moyenne mobile sur trois mois du taux de croissance du pourcentage de firmes en mauvaise situation financière et crTroc la moyenne mobile du taux de croissance du troc (en pourcentage des ventes industrielles). Les variables, issues du Russian Economic Trend et du Russian Economic Barometer, sont mensuelles.

Tableau 2. Procédure du maximum de vraisemblance de Johansen (1993-7; 1998-11)

\begin{tabular}{c|c|c|c|c}
\hline $\mathrm{H}_{0}:$ rang $=p$ & $\begin{array}{c}\text { Statistique } \\
\text { de la valeur } \\
\text { propre maximale }\end{array}$ & $\begin{array}{c}\text { Valeur critique } \\
\text { à 95\% }\end{array}$ & $\begin{array}{c}\text { Statistique } \\
\text { de la Trace }\end{array}$ & $\begin{array}{c}\text { Valeur critique } \\
\text { à 95 \% }\end{array}$ \\
\hline$p=0$ & $37,63^{* *}$ & 21 & $46,51^{* *}$ & 29,7 \\
$p \leqslant 1$ & 8,32 & 14,1 & 8,87 & 14,4 \\
$p \leqslant 2$ & 0,55 & 3,8 & 0,55 & 3,8 \\
\hline
\end{tabular}

\begin{tabular}{|c|c|c|}
\hline troc & $\begin{array}{l}\text { Vecteurs cointégrants } \\
\text { goodfin }\end{array}$ & taux d'intérêt \\
\hline 1 & 0,5224 & $-0,1422$ \\
\hline 0,3429 & 1 & 1,363 \\
\hline$-0,5096$ & 0,07341 & 1 \\
\hline
\end{tabular}

Le nombre de retards (5) a été choisi sur la base du critère d'information de Hannan-Quinn.

* significatif à $95 \%$; ** significatif à $99 \%$. 
La relation de court terme est déterminée par le modèle à correction d'erreur ${ }^{1}$ suivant :

$$
\begin{aligned}
& \Delta \text { troc }=0,13-0,19 \Delta \text { goodfin }-0,15 \Delta \text { goodfin }_{t-1}+0,11 \Delta \text { taux d'intérêt } t_{t}-0,68 \mathrm{ECM}_{t-1} \\
& (0,5)(-3,7) \quad(-2,7) \quad(2,7) \quad(-4,7) \\
& \mathrm{R}^{2}=0,40 \quad \mathrm{DW}=2,13 \quad \operatorname{AR}(5,55)=1,69 \quad \operatorname{ARCH}(5,50)=0,30 \quad \text { Normalité } \chi^{2}=2,3
\end{aligned}
$$

avec $\mathrm{ECM}_{t-1}$ le terme à correction d'erreur.

À court, comme à long terme, le troc apparaît positivement lié au niveau des taux d'intérêt réels et négativement à la situation financière des entreprises. Des tests de causalité, ainsi que des tests de variable omise (multiplicateur de Lagrange), non significatifs, concluent que d'autres variables financières, telles que les taux de liquidité, ou réelles, comme le niveau de production ou le taux d'utilisation des capacités de production, ne permettent pas d'expliquer le phénomène du troc en Russie.

Les enquêtes confirment le lien entre troc et contrainte de liquidité, mais sans préciser les contours de cette dernière. Ainsi, $72 \%$ des entreprises citent leur propre liquidité comme raison très importante du troc, $74 \%$ celle de leur partenaire, tandis que pour $49 \%$ le troc est un moyen de maintenir la production (Commander et Mumssen [1998]).

Le caractère non significatif de l'inflation ou du ratio de liquidité suggère que la contrainte de liquidité à l'origine du troc ne peut être entièrement imputée à la politique monétaire. Il convient donc d'étudier plus précisément la nature de cette contrainte à un niveau désagrégé.

\section{L'EXPLICATION DUALE DU TROC}

Le troc étant directement lié à des contraintes de liquidité, il importe d'établir si celles-ci proviennent des performances réelles des entreprises-dans ce cas le troc serait un moyen de survie pour des entreprises ne pouvant ou ne voulant pas se restructurer - ou du manque de liquidités, dans un contexte d'imperfection des marchés financiers. Le rationnement du crédit se justifie dans les économies en transition pour au moins trois grandes raisons : les créanciers font face à une forte incertitude concernant les performances futures de leurs débiteurs, les comptes sont opaques, la protection juridique des créanciers est faible. Mais le rationnement du crédit russe est surtout concomitant d'un phénomène important d'anti-sélection. Brana, Maurel et Sgard [1999] montrent que le crédit est alloué principalement aux firmes non profitables, déjà endettées, tandis que les entreprises les plus profitables sont totalement exclues du marché des fonds prêtables. Le troc permettrait à ces dernières de survivre dans ce contexte et de maintenir leur production (Linz et Krueger [1998] ; Ellingsen [1998]).

1. Toutes les variables, à l'exception du terme à correction d'erreur, sont différenciées et sont donc $\mathrm{I}(0)$. 
Notre analyse est effectuée sur des données de panel qui sont celles des enquêtes du Russian Economic Barometer. 736 entreprises ont été interrogées en juin 1995, décembre 1995, juin 1996 et décembre 1996. L'échantillon a été séparé en deux sous-ensembles selon que l'entreprise est endettée (252 entreprises) ou non (484 entreprises), de manière à prendre en compte ce phénomène d'anti-sélection. On s'attend à ce que les déterminants du troc soient différents dans les deux sous-échantillons.

L'analyse de panel permet de tenir compte d'effets fixes tels que la structure de propriété ou la taille des firmes, la région ou le type d'industrie. Il apparaît en effet (cf. Brana et Maurel [1999]) que le troc est davantage utilisé dans les secteurs plus éloignés du consommateur et dans ceux nécessitant des paiements de gros montants, comme les biens d'investissement ou d'équipement. Le troc augmente au fur à mesure que l'on s'éloigne des grandes villes (Moscou et St. Petersbourg) et plus généralement des régions de l'Est. Enfin, il est d'autant plus utilisé que la taille de la firme, mesurée par le nombre de salariés, est grande ${ }^{1}$. La principale difficulté du troc étant de trouver un partenaire d'échange, on peut penser qu'une entreprise de grande taille est plus susceptible de posséder l'investissement en capital relationnel nécessaire. D'après Aukutsionek [1998], plus une entreprise a recours au troc, plus elle a conservé des relations avec ses anciens fournisseurs. Le troc, pour une grande part, ne fait que maintenir d'anciennes relations commerciales.

Le modèle estimé est de la forme :

$$
\begin{aligned}
\text { troc }_{i t}=a+b_{1} \text { stocks }_{i t}+b_{2} \lim \text { dde }_{i t}+b_{3} \text { badfin }_{i t}+b_{4} \text { labor }_{i t}+t_{1} \mathrm{Q} 1+t_{2} \mathrm{Q} 2+t_{3} \mathrm{Q} 3+v_{i}+u_{i} \\
\Leftrightarrow \text { troc }_{i t}-\overline{\text { troc }}_{i}=b_{1}\left(\text { stocks }_{i t}-\overline{\text { stocks }}_{i}\right)+b_{2}\left(\lim d d e_{i t}-\overline{\lim d d e_{i}}\right)+b_{3}\left(\text { badfin }_{i t}-\overline{\text { badfin }}_{i}\right) \\
\quad+b_{4}\left(\text { labor }_{i t}-\overline{\text { labor }}_{i}\right)+t_{1}(\mathrm{Q} 1-\overline{\mathrm{Q} 1})+t_{2}(\mathrm{Q} 2-\overline{\mathrm{Q} 2})+t_{3}(\mathrm{Q} 3-\overline{\mathrm{Q} 3})+u_{i t}-\bar{u}_{i}
\end{aligned}
$$

avec :

- Troc, la part des ventes sous forme de troc (\%), pour chaque entreprise, à chaque période ( $t$ est successivement égal à Q1 = juin 1995, Q2 = décembre 1995, Q3 = juin 1996, Q4 = décembre 1996) et $\overline{\operatorname{troc}}_{i}=\sum_{t}$ troc $_{i t} / \mathrm{T}_{i}$;

- Stocks représente les stocks, dont le niveau «normal » pour l'entreprise et fixé à 100 et $\overline{\text { stocks }}_{i}=\sum_{t}$ stocks $_{i t} / \mathrm{T}_{i}$.

- lim dde est fixé égal à un si le principal obstacle à la production pour l'entreprise est le manque de demande. On a ${\overline{\lim d d e_{i}}}_{i}=\sum_{t} \lim d d e_{i t} / \mathrm{T}_{i}$.

- badfin prend la valeur un (l'entreprise considère que sa situation financière est bonne), 2 (situation financière normale) et 3 (sa situation financière est mauvaise). En outre, $\overline{\text { badfin }}_{i}=\sum_{t}$ badfin $_{i t} / \mathrm{T}_{i}$;

- labor est le nombre d'employés et $\overline{\text { labor }}_{i}=\sum_{t}$ labor $_{i t} / \mathrm{T}_{i}$.

- $v_{i}+u_{i t}$ est le résidu, $v_{i}$ étant le résidu unitaire spécifique et $u_{i t}$ le résidu traditionnel supposé iid;

1. Dans une économie de marché, ce sont plutôt les petites entreprises qui ont recours au troc, car ce sont généralement elles qui rencontrent le plus de problèmes de cash flow (Prendergast et Stole [1996]). 
- Q1, Q2, Q3 sont des dummies égales à un quand l'observation correspond respectivement au premier semestre (juin 1995), au second (décembre 1995), au troisième (juin 1996). Ces variables permettent de prendre en compte la croissance du troc dans le temps.

Les résultats sont regroupés dans le tableau 3.

On observe que les déterminants du troc diffèrent selon le type d'entreprise ${ }^{1}$. Pour les entreprises endettées, le recours plus fréquent au troc est associé à l'augmentation des stocks, mais ne dépend pas du fait que l'entreprise soit ou non soumise à une contrainte de débouchés (lim dde). Cela peut signifier que la production de ces entreprises est déconnectée de la demande finale, le troc étant utile pour se débarrasser des invendus (Prendergast et Stole [1996]). Le troc permettrait à ces entreprises de continuer à produire des biens « mous » pour lesquels il n'existe pas de demande effective, et de différer ainsi les restructurations (Gaddy et Ickes [1998]).

En ce qui concerne les entreprises exclues du marché du crédit, le troc apparaît d'autant plus utilisé que l'entreprise voit ses problèmes de débouchés se réduire, tandis que la variable de stocks n'est plus significative. On peut imaginer que, dans un contexte où l'entreprise n'a aucun accès au financement externe, toute hausse de la demande pose des problèmes de fond de roulement, qu'elle résout en ayant recours au troc. Sans ce dernier, le manque de liquidité de court terme serait un frein à l'augmentation de son offre de production ${ }^{2}$.

Comme au plan macroéconomique, le troc augmente avec la dégradation de la situation financière de l'entreprise (badfin) au niveau de la firme, quel que soit son niveau d'endettement. Cependant, les commentaires précédents impliquent que le terme de «situation financière » n'a pas la même signification, ni les mêmes causes, selon le type d'entreprise. Pour les firmes endettées, le troc permet de fonctionner sur les bases de l'ancien système. La situation financière est directement liée au manque de restructurations et au maintien de comportements passifs, et le troc, comme la finance externe, peut être perçu comme un moyen de survie. En revanche, pour les entreprises exclues du marché du crédit, la situation financière fait plutôt référence au manque de capital circulant, qui peut empêcher la firme de se développer. Le troc est alors un moyen de répondre à la demande, dans un contexte de rationnement du crédit.

1. La distinction entre entreprises endettées et non endettées est fondée sur l'acceptation ou non de la discipline de marché et sur l'instauration ou non de contraintes budgétaires dures. Elle ne correspond pas simplement à la distinction entre entreprises profitables/non profitables, qui existe grâce à la variable profit dans le $R E B$, mais ne permet pas d'expliquer de manière satisfaisante le troc. Ces résultats confirment que le profit n'est pas un bon indicateur du degré de restructuration et de discipline financière lorsque les réformes manquent de cohérence.

2. Le troc, le crédit commercial et le crédit bancaire sont des moyens équivalents pour offrir au producteur final du capital circulant. Mais par rapport au crédit commercial, le troc a l'avantage de supprimer le risque de crédit, ce qui est un attrait important dans un contexte où les droits des créditeurs sont faibles. Le troc a également l'avantage d'être moins coûteux que la pratique du pré paiement. 
Tableau 3. Estimations en données de panel

\begin{tabular}{c|c|c}
\hline & \multicolumn{2}{|c}{ Échantillon total } \\
\hline Troc & Effets aléatoires & Effets fixes \\
\hline stocks & 0,036 & 0,037 \\
lim dde & $-1,600$ & $-0,922$ \\
badfin & 3,517 & 0,527 \\
labor & 0,00203 & 0,00152 \\
Q1 & $-18,368$ & $-18,034$ \\
Q2 & $-13,331$ & $-13,284$ \\
Q3 & $-3,206$ & $-2,986$ \\
constante & 26,728 & \\
$\mathrm{R}^{2}$ & 0,1488 & 736 \\
\hline
\end{tabular}

Test de spécification d'Hausman

\begin{tabular}{|c|c|c|c|c|}
\hline \multirow{2}{*}{\multicolumn{2}{|c|}{$\begin{array}{c}\text { Chi( ) } \\
\text { Prob }>\text { Chi2 }\end{array}$}} & \multicolumn{3}{|c|}{$\operatorname{Chi}(7)=14,56$} \\
\hline & & \multicolumn{3}{|c|}{0,042} \\
\hline & \multicolumn{2}{|c|}{ Firmes endettées } & \multicolumn{2}{|c|}{ Firmes non endettées } \\
\hline Troc & Effets aléatoires & Effets fixes & Effets aléatoires & Effets fixes \\
\hline stocks & 0,052 & 0,077 & 0,013 & $-0,010$ \\
\hline $\lim d d e$ & 0,991 & 0,823 & $-8,022$ & $-6,575$ \\
\hline badfin & 4,674 & 1,536 & 6,043 & 1,533 \\
\hline labor & 0,00215 & 0,00050 & 0,00413 & 0,01105 \\
\hline Q1 & $-17,079$ & $-17,330$ & $-22,790$ & $-22,247$ \\
\hline Q2 & $-12,308$ & $-12,398$ & $-15,259$ & $-15,706$ \\
\hline Q3 & $-2,320$ & $-2,709$ & $-5,012$ & $-5,906$ \\
\hline constante & 19,368 & & 25,926 & \\
\hline $\mathbf{R}^{2}$ & 0,1544 & & 0,1770 & \\
\hline Nombre d'observations & \multicolumn{2}{|l|}{484} & \multicolumn{2}{|l|}{252} \\
\hline Nombre de groupes & \multicolumn{2}{|l|}{310} & \multicolumn{2}{|l|}{175} \\
\hline \multicolumn{5}{|c|}{ Test de spécification d'Hausman } \\
\hline $\mathrm{Chi}()$ & \multicolumn{2}{|c|}{$\operatorname{Chi}(7)=5,34$} & \multicolumn{2}{|c|}{$\operatorname{Chi}(6)=3,56$} \\
\hline Prob $>$ Chi2 & \multicolumn{2}{|c|}{0,618} & \multicolumn{2}{|c|}{0,8286} \\
\hline $\begin{array}{l}\text { Les coefficients en italiques } n \\
\text { Source des données : Russian }\end{array}$ & $\begin{array}{l}\text { int pas significatif } \\
\text { onomic Barometer }\end{array}$ & 995-1996]. & & \\
\hline
\end{tabular}




\section{CONCLUSION}

Le processus de transition peut être perçu comme le passage d'une économie centralement planifiée à une économie monétaire, dans laquelle la monnaie impose des contraintes budgétaires dures (Poser [1998]). Le développement important du troc, comme des substituts non monétaires peut de fait être perçu comme le signe d'un blocage du processus de transition et de restructuration. Le troc réduit la capacité de la monnaie à « durcir » les contraintes budgétaires. Il consolide les relations existantes entre firmes, réduit la concurrence et crée une demande artificielle. Les changements de prix relatifs perdent de leur pertinence. Le processus de transition est ralenti et l'évaluation des firmes devient sans fondement. Au niveau de la politique macroéconomique, le troc, en favorisant l'évasion fiscale, réduit les marges de manœuvre de la politique budgétaire. De même, le contrôle monétaire et la politique de stabilisation deviennent problématiques.

Pour certaines entreprises, le troc peut être analysé comme un moyen d'éviter les restructurations, tandis que, pour d'autres, il réduit la contrainte de liquidité dans un contexte de rationnement du crédit bancaire. La priorité doit donc être donnée à la restructuration économique, des banques comme des entreprises, ainsi qu'à la mise en place de procédures de faillite efficaces, de manière à mettre un terme au processus de désintermédiation et aux problèmes d'aléa de moralité.

\section{RÉFÉRENCES BIBLIOGRAPHIQUES}

AlfaNDARI G., SCHAFFer M.E. [1996], «Arrears' in the Russian Enterprise Sector », dans COMmANDER S., FAN, SCHAFFER M.E. (eds), Enterprise Restructuring and Economic Policy in Russia, EDI Development Studies, Washington DC, The World Bank.

AukutsioneK S. [1998], "Industrial Barter in Russia », Communist Economies and Economic Transformation, 10 (2), p. 179-188.

Brana S., MaUREL M. [1999], «Barter in Russia : Liquidity shortage versus Lack of Restructuring », CEPR Discussion Paper, $\mathrm{n}^{\circ} 2258$, octobre.

Brana S., Maurel M., SGARD J. [1999], « Enterprise Adjustment and the Role of Bank Credit in Russia : Evidence from a 420 Firm's Qualitative Survey », Comparative Economic Studies, 62 (4), hiver.

COMmander S., MumsSen C. [1998], « Understanding Barter in Russia », EBRD Working Paper, $\mathrm{n}^{\circ} 37$, décembre.

Ellingsen T. [1998], « Payments in Kind », Working Paper Series in Economics and Finance, $\mathrm{n}^{\circ} 244$, Stockholm School of Economics, juin.

ENGINEER M., BERnHARDT D. [1991], «Money, Barter, and the Optimality of Legal Restrictions », Journal of Political Economy, 99, p. 743-773.

GADDY C., ICKES B.W. [1998], « To Restructure or Not to Restructure : Informal Activities and Enterprise Behavior in Transition », Working Paper, The Davidson Institute Working Paper Series, $n^{\circ} 134$, février. 
HAYAShi F., MAtSUI A. [1994], « A Model of Fiat Money and Barter », NBER Working Paper, $\mathrm{n}^{\circ} 4919$, novembre.

Hendley K., ICKes B.W., Murrell P., Ryterman R. [1997], « Observations on the Use of Law by Russian Enterprises », Post-Soviet Affairs, 13 (1), janvier-mars, 19-41.

ICKes B.W., MurRell P., RYTERMAN R. [1997], «End of the Tunnel ? The Effects of Financial Stabilization in Russia », Post-Soviet Affairs, 13 (2), avril-juin, 105-133.

Johnson S., KAUfMan D., SHLEIFER A. [1997], « The Unofficial Economy in Transition », Brookings Papers on Economic Activity, Macroeconomics 2-97, Washington D.C., $159-240$.

LINZ S.J., KRUEGER G. [1998], «Enterprise Restructuring in Russia's Transition Economy : Formal and Informal Mechanisms », Comparative Economic Studies, 40 (2), p. 5-52.

POSER J.A. [1998], « Monetary Disruptions and the Emergence of Barter in FSU Economies », Communist Economies \& Economic Transformation, 10 (2), p. 157-177.

Prendergast C., Stole L. [1996], « Non-Monetary Exchange Within Firms and Industry », NBER Working Paper, $\mathrm{n}^{\circ}$ 5765, septembre. 